\title{
Imaging Findings in Patients with Zoster-Associated Plexopathy
}

\author{
(D)A.S. Zubair, (D) C. Hunt, (D). Watson, (D)A. Nelson, and (D) L.K. Jones Jr
}

\begin{abstract}
SUMMARY: Herpes zoster is a reactivation of the latent varicella zoster virus. Among the complications of herpes zoster is zosterassociated limb paresis. The clinical and imaging features of patients with zoster-associated limb paresis due to plexopathies (zosterassociated plexopathy) have had limited description in the literature. The Mayo Clinic patient data base was searched by diagnostic code for patients diagnosed with herpes zoster between January 1, 1996, and September 30, 2012. Patients who met the inclusion criteria for zoster-associated limb paresis or herpes zoster with MRIs obtained were reviewed. Ten patients with zoster-associated plexopathy were identified. Imaging abnormalities were found in $70 \%$ of patients. Secondary denervation changes in shoulder girdle muscles and nerve $\mathrm{T} 2$ signal hyperintensity were the most frequent abnormalities (50\%), followed by nerve enlargement (20\%). Enhancement was not evident in any cases despite early imaging in $80 \%$ of the cohort. These results demonstrate the clinical utility of MR imaging in confirming the diagnosis of zoster-associated plexopathy.
\end{abstract}

ABBREVIATIONS: HZ = herpes zoster; ZALP = zoster-associated limb paresis; ZAP = zoster-associated plexopathy

V aricella zoster virus is a DNA virus in the Herpesviridae family, which has been shown to affect only humans. Following initial infection or inoculation by the varicella zoster virus, the virus establishes latency in the sensory ganglia. ${ }^{1}$ The latent virus has the potential to reactivate later in a segmental cutaneous eruption known as herpes zoster (HZ) or shingles. ${ }^{1-3}$ The incidence of $\mathrm{HZ}$ ranges from 1.3 per 1000 person-years among young adults to 10.7 per 1000 person-years among patients older than 80 years of age. ${ }^{3}$ The diagnosis of uncomplicated HZ is generally made on clinical grounds without corroborative microbiologic testing.

Complications of $\mathrm{HZ}$ include postherpetic neuralgia, varicella zoster virus myelitis, segmental weakness, and delayed ischemic cerebral infarction due to varicella zoster virus-associated granulomatous vasculitis. These neurologic complications can appear simultaneously with the acute eruption of $\mathrm{HZ}$ or weeks to months after the rash has subsided.

The most common complication of $\mathrm{HZ}$ is postherpetic neuralgia. ${ }^{4,5}$ Postherpetic neuralgia is residual neuropathic pain that

Received February 4, 2015; accepted after revision January 25, 2017.

From the Mayo Medical School (A.S.Z.); Department of Radiology (C.H.), Division of Neuroradiology; Department of Neurology (J.W., A.N., L.K.J.); and Division of Pain Medicine (J.W.); Mayo Clinic, Rochester, Minnesota.

Please address correspondence to Lyell K. Jones Jr, MD, Mayo Clinic, Department of Neurology, 200 1st St SW, Rochester, MN 55905; e-mail: Jones.Lyell@mayo.edu; @LyellJ

三 Indicates article with supplemental on-line table.

http://dx.doi.org/10.3174/ajnr.A5149 lasts $\geq 3$ months and is seen in roughly $20 \%$ of all patients with $\mathrm{HZ}$ and up to one-third of patients with $\mathrm{HZ}$ who are older than 80 years of age. ${ }^{6,7}$

Zoster-associated limb paresis (ZALP) is another recognized complication of $\mathrm{HZ}$ and consists of segmental weakness in a limb affected by HZ. ${ }^{8-10}$ Many questions remain about ZALP, including the precise localization and mechanism of the motor abnormalities and the pathophysiology and imaging findings of the condition. ${ }^{8,9}$ The clinical and imaging features of patients with ZALP due to plexopathies (zoster-associated plexopathy [ZAP]) have had limited description in the literature. ${ }^{11-14}$ There are few dedicated reports of imaging findings in patients with ZAP despite imaging playing a crucial role in the localization of lesions, excluding other etiologies, and determining the extent of the lesions causing ZAP. ${ }^{15}$

The aim of this study was to identify the specific clinical and imaging features and their frequencies in patients with zosterassociated plexopathy.

\section{MATERIALS AND METHODS \\ Case Series}

This study was approved by the institutional review board and conducted at the Mayo Clinic with a waiver of informed consent being obtained before the study. The Mayo Clinic patient data base was searched by diagnostic code for patients diagnosed with herpes zoster between January 1, 1996, and September 30, 2012. Patients clinically diagnosed with ZAP who had MR imaging of 
the affected plexus were included. The case definition for ZAP was the following: 1) documented history of examination evidence of a cutaneous vesicular eruption consistent with HZ; 2) evidence of temporal (within 30 days) and geographic (same limb) associated weakness confirmed on examination by a Mayo Clinic neurologist; and 3) electrodiagnostic evaluation confirming localization to the brachial or lumbosacral plexus during the symptomatic period of weakness. This case definition uses the temporal relationship between the HZ eruption and the limb weakness to establish the ZAP diagnosis; as in prior series, the typically delayed patient presentation limits the utility of virologic or serologic biomarker confirmatory testing. ${ }^{16}$ Descriptive statistics were applied to the findings.

\section{Clinical Localization}

All patients underwent neurologic examinations administered by a board-certified Mayo Clinic neurologist. The examination findings were reviewed for severity and the distribution of weakness, sensory loss, and changes in muscle stretch reflexes. Plexopathy severity was determined by a standardized scoring of muscle weakness. $^{8}$

\section{Electrodiagnostic Evaluations}

Electrodiagnostic studies performed on affected segments were reviewed, and the findings were summarized. Electrodiagnostic studies included nerve-conduction studies performed in standard fashion by using surface-recording electrodes. Needle electromyography was performed with a standard concentric needle electrode and included examination of at least 5 muscles in each affected segment.

\section{Imaging}

MR imaging studies of affected areas were reviewed by a boardcertified neuroradiologist (C.H.), blinded to the results of the original interpretation, which was performed by a board-certified neuroradiologist or musculoskeletal radiologist, with consensus agreement from the other authors on the imaging findings. While our current brachial plexus imaging protocols have transitioned to $3 \mathrm{~T}$ imaging, due to the time span of this study, 3 of 8 patients were imaged at only 1.5T. All of the patients with ZAP localized to the lumbosacral plexus were imaged at $1.5 \mathrm{~T}$. All studies included both pregadolinium T1- and T2-weighted images in axial, coronal, and sagittal planes. All T2-weighted sequences had either conventional fat saturation or a short tau inversion recovery sequence. Gadolinium-enhanced T1-weighted images (spoiled gradient-recalled acquisition) in at least 2 planes (axial and typically sagittal) were also obtained. Our typical protocol can be completed within 90 minutes from the time the patient enters the MR imaging suite. Root, plexus, or peripheral nerve imaging findings were classified as abnormal when associated with qualitatively prolonged nerve $\mathrm{T} 2$ or $\mathrm{T} 2{ }^{\star}$, nerve enlargement, or postgadolinium nerve enhancement based on comparison with other neural structures within the imaging field. Diffusion sequences were not applied. Contralateral structures were included in the FOV for lumbosacral plexus imaging, but contralateral brachial plexus imaging was not routinely performed. In addition, the proximal
Clinical features of patients with zoster-associated plexopathy

\begin{tabular}{ll}
\hline \multicolumn{1}{c}{ Features } & \\
\hline Mean age at onset (yr) & 74.5 (range, 54-88) \\
Men & 7 of $10(70 \%)$ \\
Upper limb affected & 8 of $10(80 \%)$ \\
Mean interval between rash & 18 \\
and weakness (days) & \\
Neuralgia 1 mo after rash & 10 of $10(100 \%)$ \\
Neuralgia 3 mo after rash & 7 of $10(70 \%)$ \\
Corticosteroid treatment & 6 of $10(60 \%)$ \\
Diabetes mellitus & 2 of $10(20 \%)$ \\
Immunosuppression & 1 of $10(10 \%)$ \\
\hline
\end{tabular}

shoulder muscles within the FOV were also examined for evidence of abnormal $\mathrm{T} 2$ signal.

\section{RESULTS}

Ten patients satisfying the case definition were identified and reviewed. Patient features are summarized in the Table, and clinical and radiographic details are outlined in the On-line Table. Eight patients $(80 \%)$ had brachial plexopathies, with the remainder diagnosed with lumbosacral plexopathies. Electrodiagnostic evaluations confirmed the localization to the brachial plexus or lumbosacral plexus in each case (On-line Table). Three patients underwent CSF examination, only 1 of which was performed within 1 month of symptom onset (patient 9, demonstrating an elevated protein level of $159 \mathrm{mg} / \mathrm{dL}$, elevated nucleated cell count of 189 cells/microliter, and a polymerase chain reaction positive for varicella). Six patients (60\%) received steroids at some point in the management of their presentation. Five of these patients received short courses of oral prednisone, all $>1$ month before evaluation, and 1 patient received high-dose IV methylprednisolone for 3 days after imaging was performed, making it unlikely that the imaging findings in this series were influenced by the steroid treatment. There were no reported complications of steroid treatment in these patients such as zoster dissemination.

MR imaging findings were abnormal in 7/10 patients (70\%). Five patients $(50 \%)$ demonstrated increased nerve T2 signal, 2 patients (20\%) demonstrated nerve enlargement, and no patients demonstrated nerve enhancement. Denervation changes were noted in 5 patients $(50 \%)$, primarily in shoulder girdle muscles included in the FOV. All except 1 of the patients with denervation changes also demonstrated abnormal nerve imaging findings; and correspondingly, all except 2 of the patients with abnormal nerve imaging findings also demonstrated denervation changes. The most commonly affected muscles were the infraspinatus (4/5), supraspinatus $(3 / 5)$, teres minor $(1 / 5)$, subscapularis $(1 / 5)$, and the deltoid (1/5). Characteristic imaging abnormalities are shown in Figs $1-3$.

\section{DISCUSSION}

This study describes the imaging abnormalities in the largest series of patients with ZAP to date. ${ }^{1-13}$ ZALP, which may localize to the root, plexus, or more peripheral nerve, is difficult to localize clinically in part because the involved myotomes often do not correspond to the dermatomes affected by the rash. ${ }^{15}$ Thus, imaging can be a very useful tool in the identification and localization of the lesions in these patients. 


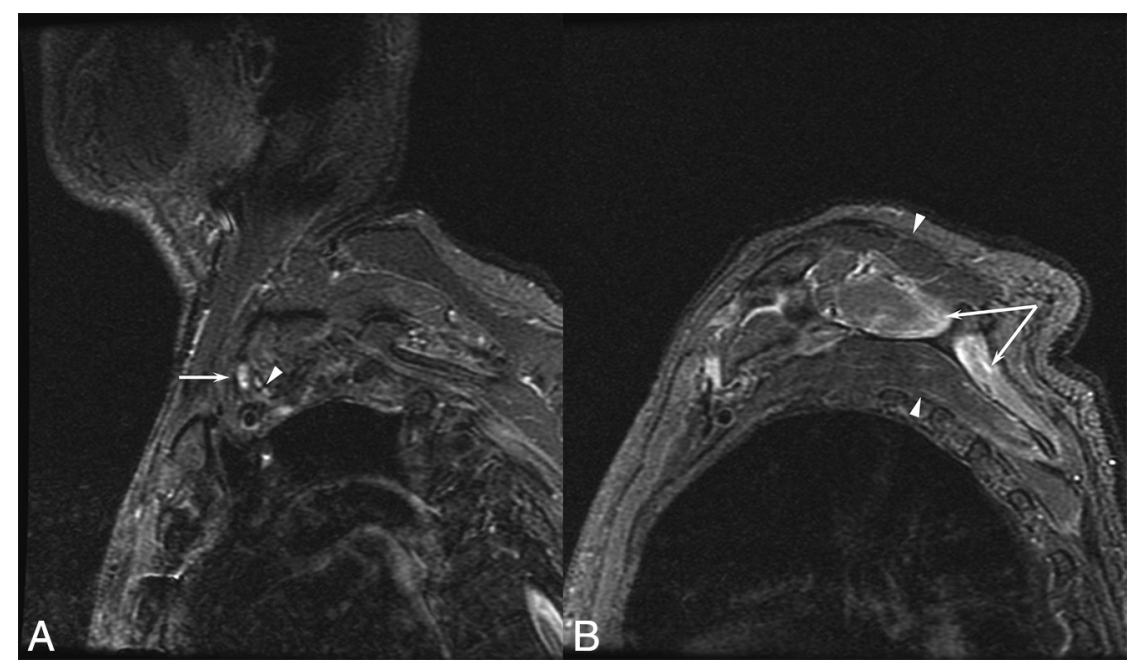

FIG 1. A 77-year-old man with a left brachial zoster-associated plexopathy. A, Sagittal inversion recovery image demonstrates increased T2 signal in the upper trunk (arrow) compared with the other elements of the plexus (arrowhead). B, Prolonged T2 is noted in the supraspinatus and infraspinatus (arrows), corresponding to denervation resulting from the plexopathy.

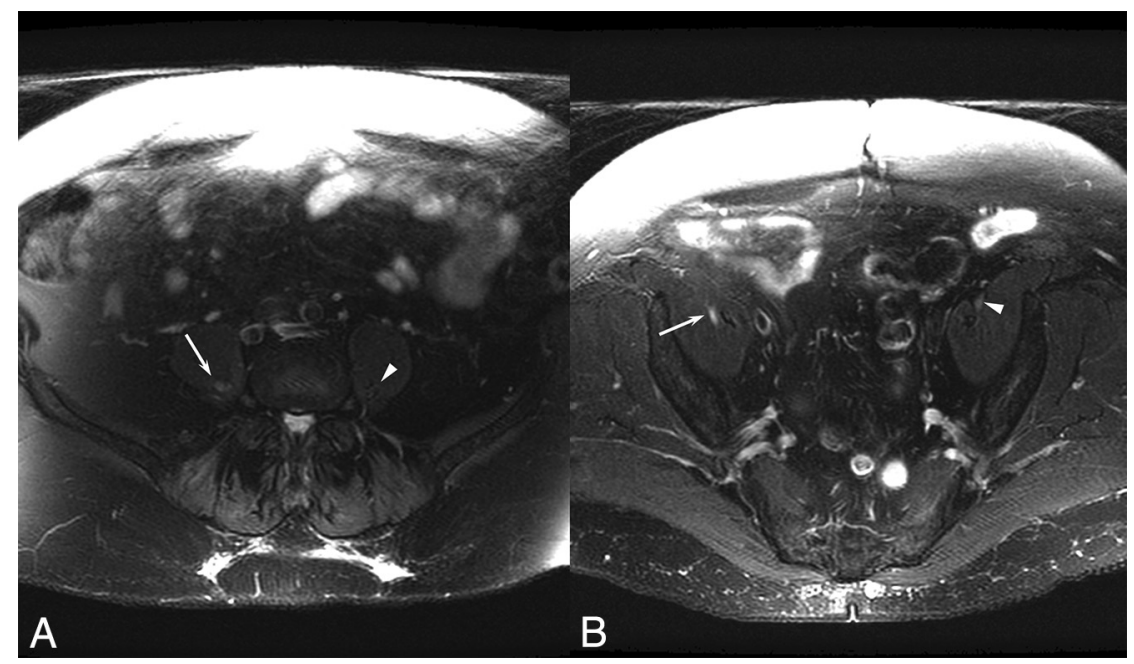

FIG 2. A 70-year-old woman with a right lumbar zoster-associated plexopathy. A, Axial T2-weighted image demonstrates increased T2 signal in the right lumbar plexus (arrow) compared with the left lumbar plexus (arrowhead). B, Increased T2 is also noted more distally in the right femoral nerve (arrow) compared with the unaffected left side (arrowhead).

Prior reports described imaging findings ranging from normal to abnormal, similar to those described in this series. The spectrum of findings in this series ranged from normal to increased T2 signal in the plexus, nerve enlargement, and denervation changes in the muscles innervated by the plexus.

The imaging findings included not only nerve abnormalities but also secondary denervation changes in affected muscles, which were seen in half of all patients. These changes were only present in the shoulder girdle muscles among those patients with brachial ZAP. This constellation of findings can be used clinically to help diagnose patients with ZAP and categorize the severity of their conditions. While no patients with ZAP in this series demonstrated nerve enhancement after administration of gadolinium contrast, postcontrast imaging may still be useful in patients with plexopathy to exclude other potential causes of weakness such as peripheral nerve tumors.

Clinically, patients with ZAP have a high rate of postherpetic neuralgia. At 1 month after the rash, 100\% of patients had neu- ralgia, and at 3 months, $70 \%$ of patients had postherpetic neuralgia. This rate exceeds previously reported rates of postherpetic neuralgia in patients with HZ (generally $20 \%-40 \%$ ), ${ }^{3,10}$ possibly attributable to more severe disease or other unrecognized factors. The rate of postherpetic neuralgia in this series was similar to that reported in all patients with ZALP. ${ }^{8}$ In addition to the need to recognize the high rate of postherpetic neuralgia in patients with ZAP, our findings also suggest that these patients are frequently typically treated with short courses of corticosteroids. While the size and structure of this series does not allow comment on efficacy, no patients experienced reported adverse effects related to the steroid treatments, such as zoster dissemination or worsening of neurologic deficits. These findings provide some evidence of safety if future corticosteroid trials are entertained in this group of patients.

The pathophysiology of ZALP due to ZAP is not completely understood. Electrophysiologic and neuropathologic studies have implied viral spread of inflammation from the dorsal root gan- 


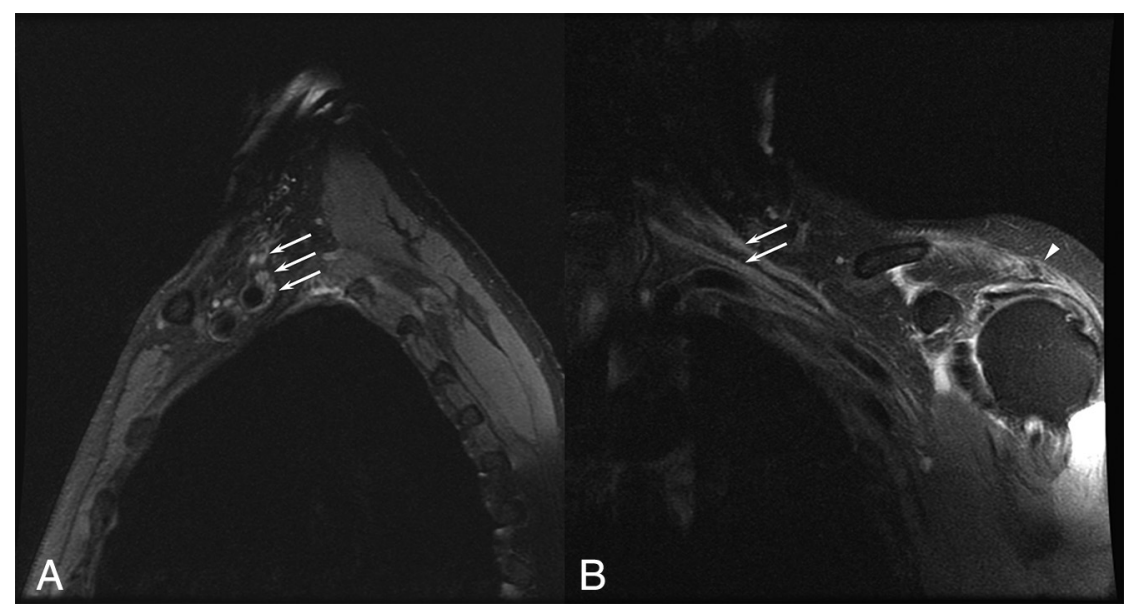

FIG 3. A 75-year-old man with a left brachial zoster-associated plexopathy. A, Sagittal T2-weighted image with fat saturation demonstrates diffusely increased T2 in the upper, middle, and lower trunks of the brachial plexus (arrows). B, Coronal T2-weighted image with fat saturation demonstrates increased T2 in the upper and middle trunks of the brachial plexus (arrows). Prolonged T2 associated with denervation is noted in the deltoid (arrowhead).

glion to adjacent nervous tissue, including the anterior horn cell and motor roots. ${ }^{12,16-18}$ Previous histologic studies of patients with $\mathrm{HZ}$ paresis indicated diffuse chronic inflammation of all trunks of the brachial plexus with destruction of myelin and axonal sparing. ${ }^{12}$ In practice, the clinical findings in the setting of a history of characteristic rash raise the suspicion of ZALP, and electrodiagnostic abnormalities that localize to the corresponding plexus confirm the presence of a plexopathy. When the diagnosis of ZAP is suspected, imaging can play an important role in supporting the diagnosis and excluding other causes of plexopathy, the primary reason in this series for performing imaging studies. While most of the imaging in this study was performed with 1.5T MR imaging, with the continued transition to 3T scanners, it is anticipated that the sensitivity of plexus MR imaging, especially of small nerves, will increase.

This study is limited by its small size and retrospective design. While our findings suggest that imaging is a useful adjunct in the evaluation of patients with ZAP, corroboration with standardized imaging protocols in larger groups of patients would be helpful, especially in the absence of specific imaging or serologic findings.

\section{CONCLUSIONS}

ZALP can result from brachial or lumbosacral plexopathies (ZAP). Patients with ZAP have a high rate of postherpetic neuralgia (100\% at 1 month, $70 \%$ at 3 months). MR imaging is a useful diagnostic technique in patients with ZAP, with $70 \%$ of patients in this series demonstrating radiographic abnormalities attributable to ZAP. Hopefully, these findings and those of future series will be useful in the diagnosis and management of patients with ZAP.

\section{REFERENCES}

1. Zerboni L, Sen N, Oliver SL, et al. Molecular mechanisms of varicella zoster virus pathogenesis. Nat Rev Microbiol 2014;12:197-210 CrossRef Medline

2. Schmidbauer M, Budka H, Pilz P, et al. Presence, distribution and spread of productive varicella zoster virus infection in nervous tissues. Brain 1992;115(pt 2):383-98 CrossRef Medline

3. Yawn BP, Saddier P, Wollan PC, et al. A population-based study of the incidence and complication rates of herpes zoster before zoster vaccine introduction. Mayo Clin Proc 2007;82:1341-49 CrossRef Medline
4. Gnann JW Jr, Whitley RJ. Clinical practice. Herpes zoster. N Engl J Med 2002;347:340-46 CrossRef Medline

5. Jung BF, Johnson RW, Griffin DR, et al. Risk factors for postherpetic neuralgia in patients with herpes zoster. Neurology 2004;62:1545-51 CrossRef Medline

6. Alshekhlee A, Tay E, Buczek M, et al. Herpes zoster with motor involvement: discordance between the distribution of skin rash and localization of peripheral nervous system dysfunction. J Clin $\mathrm{Neu}$ romuscul Dis 2011;12:153-57 CrossRef Medline

7. Cockerell OC, Ormerod IE. Focal weakness following herpes zoster. J Neurol Neurosurg Psychiatry 1993;56:1001-03 CrossRef Medline

8. Jones LK Jr, Reda H, Watson JC. Clinical, electrophysiologic, and imaging features of zoster-associated limb paresis. Muscle Nerve 2014;50:177-85 CrossRef Medline

9. Reda H, Watson JC, Jones LK Jr. Zoster-associated mononeuropathies (ZAMs): a retrospective series. Muscle Nerve 2012;45:734-39 CrossRef Medline

10. Gilden DH, Kleinschmidt-DeMasters BK, LaGuardia JJ, et al. Neurologic complications of the reactivation of varicella-zoster virus. N Engl J Med 2000;342:635-45 CrossRef Medline

11. Eyigor S, Durmaz B, Karapolat H. Monoparesis with complex regional pain syndrome-like symptoms due to brachial plexopathy caused by the varicella zoster virus: a case report. Arch Phys Med Rehabil 2006;87:1653-55 CrossRef Medline

12. Fabian VA, Wood B, Crowley P, et al. Herpes zoster brachial plexus neuritis. Clin Neuropathol 1997;16:61-64 Medline

13. Mullins GM, O'Sullivan SS, Neligan A, et al. Non-traumatic brachial plexopathies, clinical, radiological and neurophysiological findings from a tertiary centre. Clin Neurol Neurosurg 2007;109:661-66 CrossRef Medline

14. Yoleri O, Olmez N, Oztura I, et al. Segmental zoster paresis of the upper extremity: a case report. Arch Phys Med Rehabil 2005;86: 1492-94 CrossRef Medline

15. Choi JY, Kang CH, Kim BJ, et al. Brachial plexopathy following herpes zoster infection: two cases with MRI findings. J Neurol Sci 2009;285:224-26 CrossRef Medline

16. Ismail A, Rao DG, Sharrack B. Pure motor herpes zoster induced brachial plexopathy. J Neurol 2009;256:1343-45 CrossRef Medline

17. Jeevarethinam A, Ihuoma A, Ahmad N. Herpes zoster brachial plexopathy with predominant radial nerve palsy. Clin Med (Lond) 2009; 9:500-01 CrossRef Medline

18. Ayoub T, Raman V, Chowdhury M. Brachial neuritis caused by varicella-zoster diagnosed by changes in brachial plexus on MRI. $\mathrm{J} \mathrm{Neu}$ rol 2010;257:1-4 CrossRef Medline 